N. E. Piskunov, W. W. Weiss, D. F. Gray, eds.

\title{
Dynamical Behavior of the Upper Solar Photosphere
}

\author{
Robert J. Rutten \\ Sterrekundig Instituut, Utrecht University \\ Postbus 80000, NL 3508 TA Utrecht, The Netherlands
}

\begin{abstract}
The dynamical behavior of upper cool-star photospheres constitutes the next step in realistic atmosphere modeling after the successful numerical implementation of low-photosphere granulation. The solar example shows that this behavior is complex even when magnetism is ignored. Acoustic waves are a principal ingredient, but so are atmospheric gravity waves. Acoustic events provide less pistoning than expected. Correlation and Fourier analyses of image sequences from TRACE demonstrate the ubiquity of gravity waves.
\end{abstract}

\section{Introduction: the next modeling arena}

I address the dynamics of the solar photosphere within the context of this meeting, i.e., taking the solar atmosphere as cool-star archetype observed with sufficient spatio-temporal resolution to diagnose dynamical processes at their intrinsic scales. I concentrate on the upper photosphere in quiet internetwork areas, on the unproven premise that hydrodynamical phenomena dominate there over magnetism-related dynamism, and in the expectation that this domain represents the next arena for realistic cool-star atmosphere modeling - where "realistic" implies relaxing the drastic simplification of of plane-parallel equilibria by including actual dynamical processes as diagnosed initially in the solar atmosphere. At low-photosphere levels this is successfully accomplished for solar-like stellar granulation (cf. Asplund's contribution); it is now time to move upward. In terms if hydrodynamics it means including acoustic and gravity wave excitation and interference in cool-star atmosphere modeling. The next major step will be to add magnetic network and network canopies (cf. Title's contribution).

\section{The internetwork scene}

Figure 1 portrays the internetwork arena I discuss here. After my presentation Alan Title admonished me sternly to "never show that cartoon again because it is all wrong". He meant that its rendering of the network field topology is oversimplified, as it is indeed. Real network fields possess very complex threedimensional topologies having a large variety of connections over a wide range of scales, a full domain of connectivity rather than simple bipolar bar magnet or unipolar helmet ("wine glass") geometry. Defining the magnetic canopy correctly as the $\beta=1$ pressure-equality surface makes it not follow any particular field line, but a very contorted and time-variant surface. The magnetic carpet 


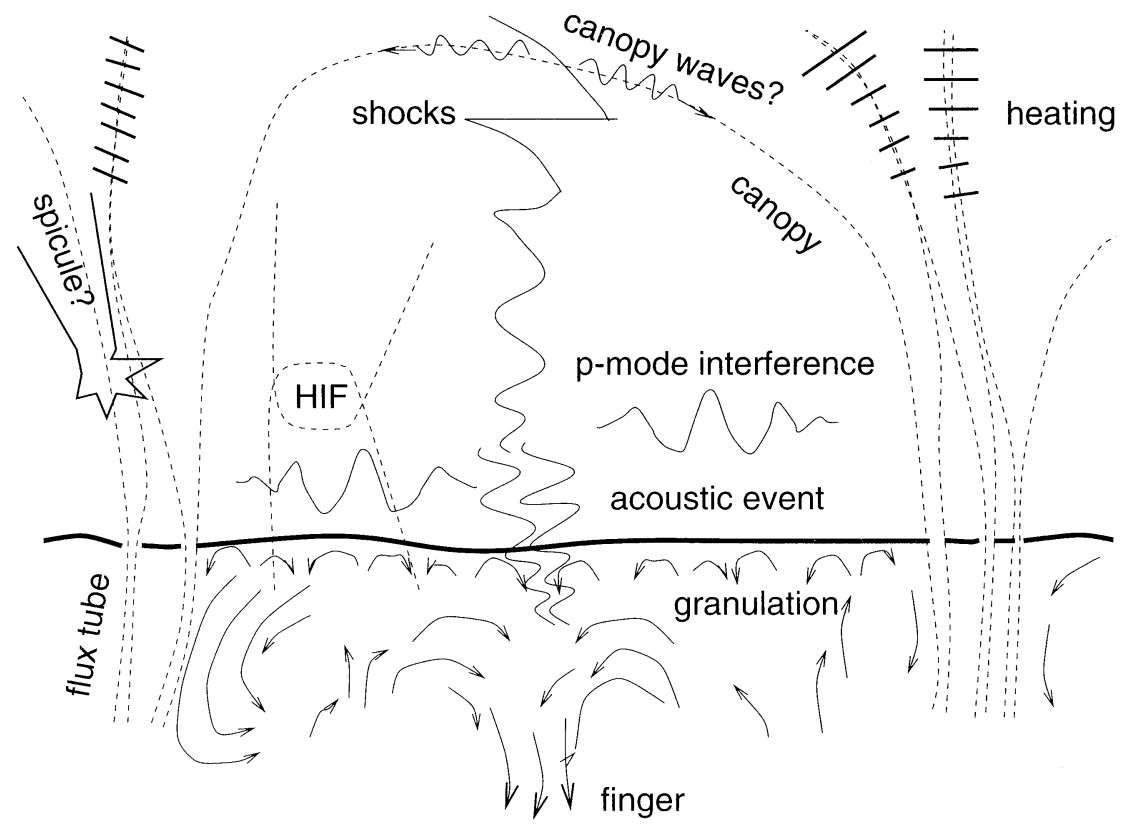

Figure 1. The solar internetwork scene as a cartoon representing a vertical cut through the lower solar atmosphere containing one supergranulation cell and bipolar network on its borders. The thick line indicates the $\tau=1$ optical surface, dashed curves indicate selected magnetic field lines, subsurface arrows convective flows, wavy curves acoustic oscillations. "HIF" stands for horizontal internetwork fields as described by Lites et al. (1996). "Canopy" defines the surface where magnetism starts dominating. The convection converges into downflow fingers as computed by Stein \& Nordlund (e.g., 1989, 2000). "Acoustic events" are sound-emitting downflows as computed by Skartlien et al. (2000). Acoustic waves run upward and steepen into shocks as computed by Carlsson \& Stein (1997). At the canopy they tend to be reflected and converted into magnetic modes as observed by McIntosh et al. (2001) and computed by Rosenthal et al. (2002). Spicules were entered with the separatrix geometry of van Ballegooijen \& Nisenson (1999) in mind. Network heating was drawn following the whiplash shear amplification mechanism of van Ballegooijen et al. (1998). The observational fact that such heating tends to be confined to and favor denser field patches is attributed by Schrijver \& Title (2002) to the absence of reconnection-inviting null points and the restricted propagation of field braiding and twisting in the much more complex actual topology of real solar network fields. From Rutten (1999). 
is neither a combed one nor a static one (cf. Schrijver \& Title 2002, or inspect a high-resolution $\mathrm{H} \alpha$ movie).

However, in this contribution I address only the dynamical phenomena under the carpet, and additionally ignore HIF's and other internetwork fields. This severe simplification is designed to meet the modeler's need for numerical tractability. The cartoon then suggests (i) that above the convection and below the canopy acoustics is all there is that one needs to model, and (ii) that acoustic shock excitation is dominated by acoustic events. Both notions are widely held (so far also by me, cf. Rutten 1999); both turn out to be wrong.

\section{Acoustic pistoning}

The term "acoustic events" or "seismic events" was introduced by Goode and coworkers (Goode et al. 1992, Restaino et al. 1993, Rimmele et al. 1995, Goode et al. 1998, Strous et al. 2000) to denote locations where excessive acoustic flux, mostly directed downward below the surface but also upward above the surface, is excited at locations where small granules collapse from the loss of an upward sustaining flow, as modeled by Rast (1999) and Skartlien et al. (2000). The events are believed to represent major pistoning sources for the global $p$-mode oscillations (Strous et al. 2000) and also to cause bright internetwork grains in the upper photosphere by pistoning upward traveling waves (Goode 2002).

The latter are observed as $\mathrm{Ca}_{\mathrm{IIK}} \mathrm{IV}$ and $\mathrm{H}_{2 V}$ brightenings at visible wavelengths and as very similar brightenings in $1600 \AA$ and $1700 \AA$ filtergrams from TRACE (cf. Krijger et al. 2001).

In a recent paper (Hoekzema, Rimmele \& Rutten 2002) we have quantified the spatio-temporal coincidence between acoustic events and Ca II $\mathrm{K}_{2 V}$ internetwork grains using filtergram sequences from the NSO/Sacramento Peak Dunn Solar Telescope. The acoustic flux was measured from two-dimensional Dopplershift maps sampling multiple heights in the low photosphere obtained from narrow-band Universal Birefringent Filter images stepping through the Fe I $5434 \AA$ line. Simultaneous Ca II $\mathrm{K}_{2 V}$ images sampling the upper-photosphere ${ }^{1}$ brightness served to measure brightness oscillation amplitudes in internetwork areas.

Figure 2 summarizes the conclusion in the form of co-location scatter diagrams in a format developed by Strous (1994). They show a highly significant but very slight correlation between excessive acoustic flux (large abscissa values) and excessive CaII $\mathrm{K}_{2 V}$ brightness (large ordinate values). The correlation is highly significant because the sampling statistics are very high. Quantification of the co-location probability using Hoekzema's correspondence method (Hoekzema et al. 1998) confirms that for the $5 \%$ bin of pixels with maximum upward flux the likelihood to coincide with the $5 \%$ bin of maximum $\mathrm{K}_{2 V}$ oscillation amplitude is nearly twice the random one. However, the correlation is only

\footnotetext{
${ }^{1}$ The onset of the solar chromosphere is a matter of definition. It used to start at the temperature minimum but the location of the latter is at least in dispute if not highly variable in reality. I prefer to define the $\beta=1$ surface as both the magnetic canopy and the onset of the chromosphere. This surface is surely heavily warped and time-variable.
} 

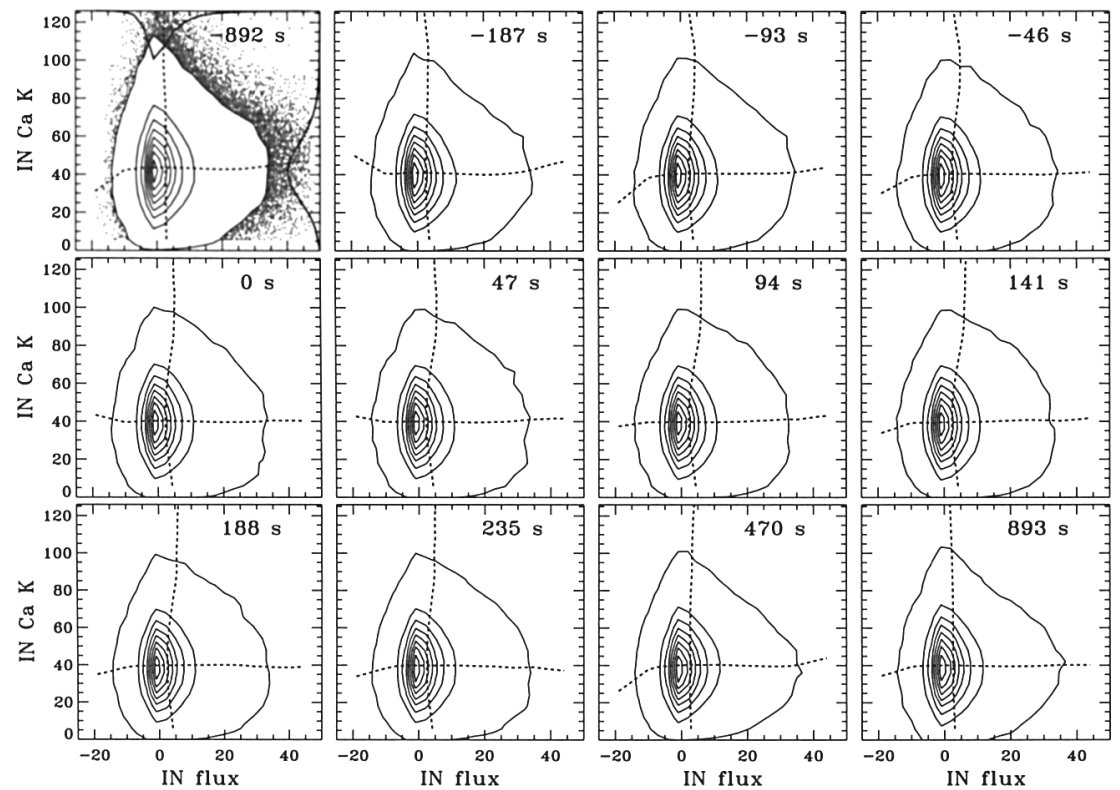

Figure 2. Time-delay scatter density plots for acoustic flux and Ca II $\mathrm{K}_{2 V}$ brightness, exclusively for internetwork (IN) areas. Each panel compares the two quantities per internetwork pixel from 20 successive simultaneous image pairs at different time delay $\Delta t$ (specified per panel, increasing along rows from top left to bottom right) between the two sequences, positive $\Delta t$ denoting $\mathrm{Ca}$ II $\mathrm{K}_{2 V}$ sampling after acoustic flux sampling. Each plot shows the pixel-by-pixel comparison but point density contours are plotted instead of single points to avoid overcrowding. Only the first panel shows those actual scatter points that fall outside the outer contour. The solid curves along the top and righthand axes of the first panel show the occurrence distributions on inverted scales. Both have extended large-value tails. The dashed curves are the first moments of the point density distribution per bin in each quantity. Their perpendicularity in the first and last panels indicates complete absence of correlation between flux amplitude and $\mathrm{Ca}$ II $\mathrm{K}_{2 V}$ brightness at long time delay between the two. The rightward bending of the upper part of the vertical moment curve indicates small but significant correlation between extreme internetwork $\mathrm{K}_{2 V}$ brightness and large upward acoustic flux during $\Delta t \approx-1$ to $+5 \mathrm{~min}$. From Hoekzema et al. (2002).

slight and very far from one-to-one correspondence (twenty times the random chance for a $5 \%$ bin).

Thus, some of the more excessive flux events indeed poke through into the upper photosphere to cause excessive internetwork grains, but only very few do so noticeably. 


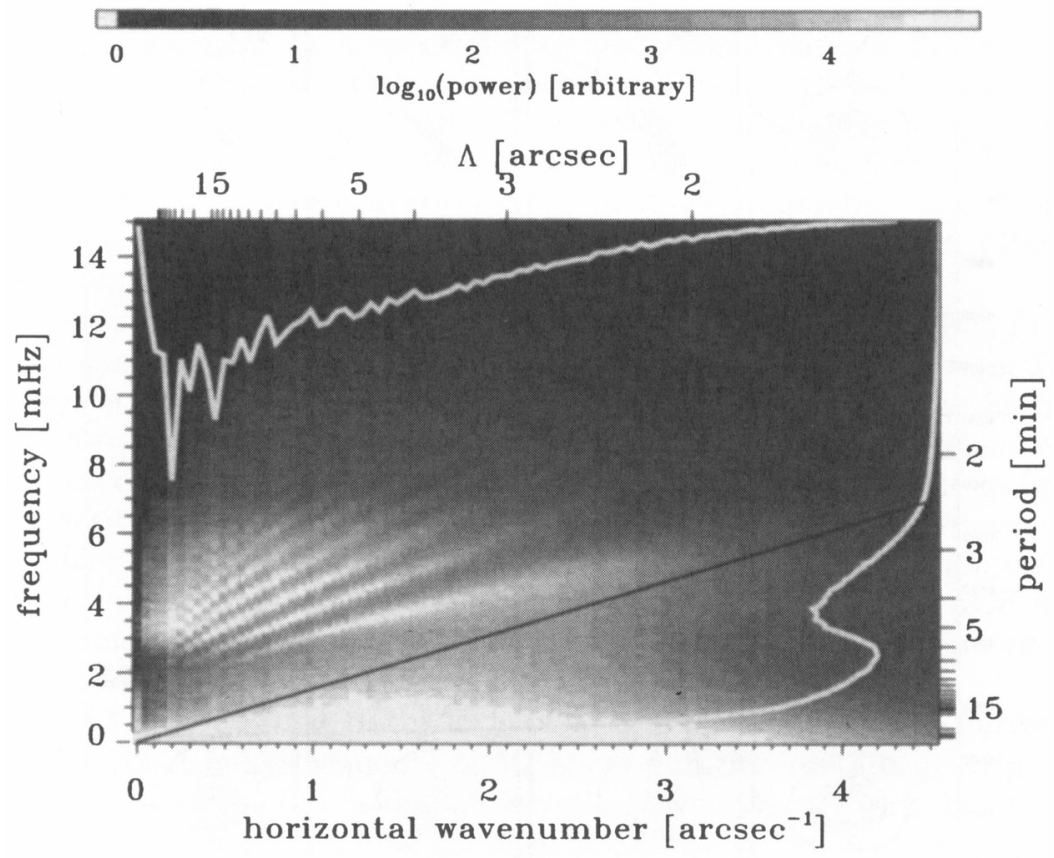

Figure 3. Upper-photosphere $\left(k_{h}, f\right)$ power spectrum from TRACE $1700 \AA$ images. Axes: horizontal wavenumber $k_{h}$ and temporal frequency $f$. The corresponding wavelengths and periodicities are specified along the top and the righthand sides. Greyscale: modulation power as specified in the bar above the plot. The white curves along the sides are the temporal and spatial means, on linear scales in arbitrary units. The slanted black line is the Lamb line $f=(1 / 2 \pi) c_{s} k_{h}$ for $c_{s}=7.2 \mathrm{~km} \mathrm{~s}^{-1}$. The column structure is caused by TRACE data compression. From Rutten \& Krijger (2002).

\section{Atmospheric gravity waves}

The low spatio-temporal correlation between acoustic events in the lower photosphere and large-amplitude brightness modulation in the upper photosphere suggests that another agent is at work. Figures $4-5$ identify that other agent as atmospheric gravity waves. They are taken from Rutten \& Krijger (2002) which gives the full story. It is summarized here.

The early work of e.g., Whitaker (1963), Lighthill (1967), Stein (1967) and Schmieder (1977) and the beautiful studies of Mihalas \& Toomre $(1981,1982)$ have shown that gravity waves should be copiously excited by convective overshoot and should be abundantly present in the upper photosphere and low chromosphere up to their breaking height, but that their detection is difficult because their long periods and short wavelengths are comparable to and hard to disentangle from granulation. Also, since they propagate preferentially in near-horizontal directions, they produce only small radial Dopplershifts and quickly move off 


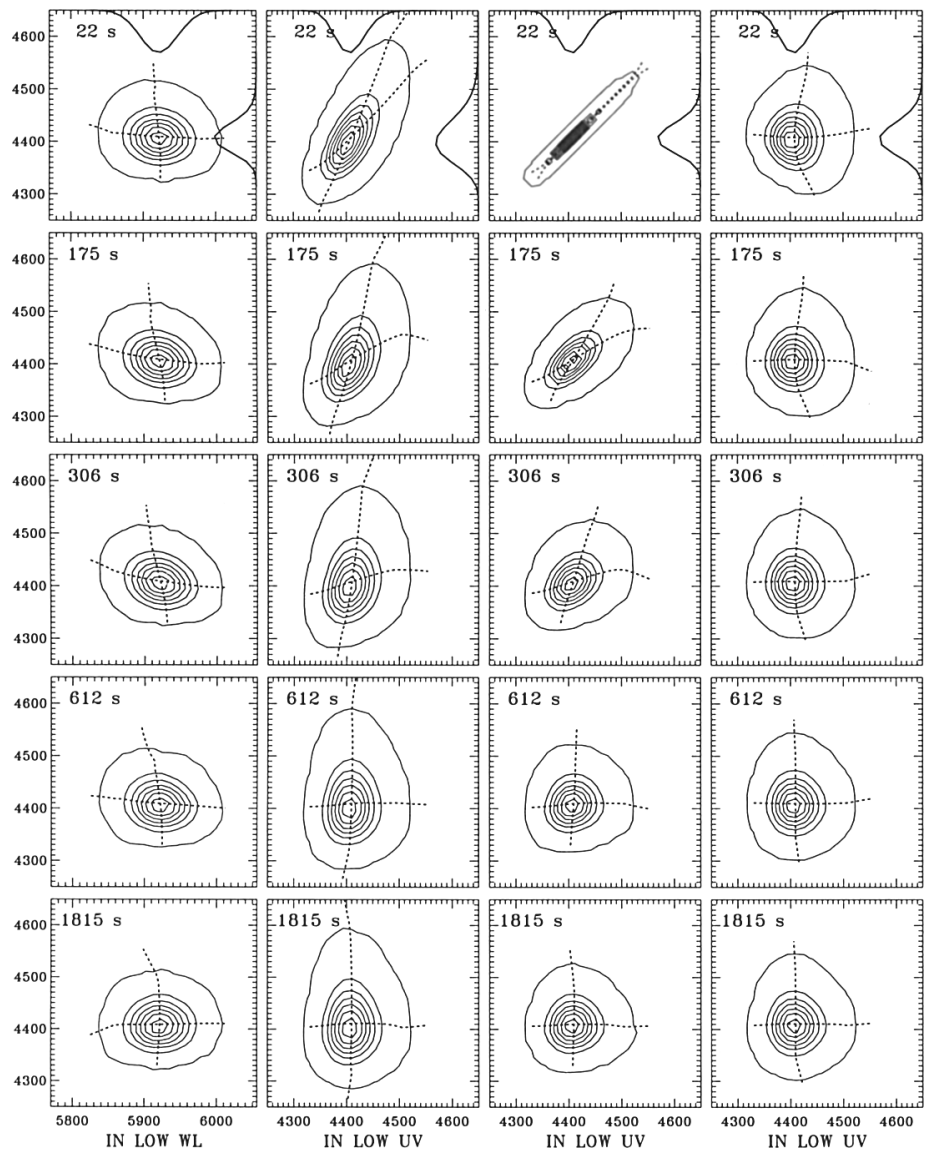

Figure 4. Time-delay scatter diagrams from TRACE image sequences, for internetwork (IN) areas only and in the format of Fig. 2. Each individual sample point specifies brightness temperature $T_{b}(x, y, t)$ (horizontal) and time-delayed brightness temperature $T_{b}(x, y, t+\Delta t)$ (vertical) for one pixel $(x, y)$. Sample density contours rather than individual samples are plotted to avoid plot saturation. The time delays $\Delta t$ are specified in each panel and increase from top to bottom. The solid curves in the top panels show the occurrence distributions of the quantities plotted along $x$ and $y$ on inverted normalized scales. The dashed curves show the first moments of the sample density distributions per $x$ and $y$ bin, i.e., the locations of the centers of gravity along $x$ and $y$ cuts. First column: time-delayed low-pass filtered $1700 \AA$ brightness temperature $\mathrm{UV}(t+\Delta t)$ against low-pass filtered white-light brightness temperature WL $(t)$. Second column: unfiltered UV against low-pass UV. Third column: low-pass UV against low-pass UV. Fourth column: high-pass UV against low-pass UV, with the mean UV internetwork value $\left(T_{\mathrm{b}}=4418 \mathrm{~K}\right)$ added to the high-pass $\mathrm{UV}$ modulation for $y$-axis compatibility. From Rutten \& Krijger (2002). 
a high-resolution pixel or a spectrometer slit. The observational evidence for their existence was so far rather inconclusive (Frazier 1968, Schmieder 1976, Cram 1978, Brown \& Harrison 1980, Durrant \& Nesis 1981, Staiger et al. 1984, Staiger 1987, Deubner \& Fleck 1989, Bonet et al. 1991, Komm et al. 1991), with the most convincing demonstration given in the wavenumber- and frequencyresolved $\left(k_{h}, f\right)$ phase-difference spectra of Kneer \& von Uexküll (1993) and Straus \& Bonaccini (1997).

Recently, gravity waves showed up rather clearly in similar $\left(k_{h}, f\right)$ phasedifference spectra derived from $1700 \AA$ and $1600 \AA$ image sequences from TRACE by Krijger et al. (2001). Ultraviolet image sequences are perhaps the best diagnostic for gravity waves because they sample intensity rather than velocity (gravity waves primarily affect the temperature), sample them high up (gravitywave intensity modulation grows steeply with height), permit two-wavelength phase-difference evaluation over relatively small height of formation difference (so that a slanted wave remains on-pixel between the two) and TRACE has just sufficient angular resolution (1 arcsec). In addition, TRACE offers co-spatial white light imaging at the same resolution.

Figure 3 sets the stage in the form of a $\left(k_{h}, f\right)$ power spectrum derived from a TRACE $1700 \AA$ image sequence of 3.7 hours duration (details in Krijger et al. 2001). It holds for the full field of observation including network, but the latter was so quiet that this figure may be taken as representative of internetwork only. The spatially averaged temporal power spectrum at right shows a high wide peak representing the three-minute oscillation (often called "chromospheric" but it is mostly an under-the-canopy phenomenon, cf. Rosenthal et al. 2002), which is resolved in $p$-mode ridges and pseudo-ridges at left. At slower periodicities there is a gradual increase toward much higher power. Its spatial decomposition along the $x$-axis shows large power already from granular scales onward. Thus, below the Lamb line there is much long-period power at roughly mesogranular scales.

Figure 4 analyses the nature of this small-scale long-period power through co-alignment analyses using the Strous scatter plot format introduced in Fig. 2, again only for internetwork pixels. The first column shows time-delay scatter diagrams between $1700 \AA$ (UV) brightness and white-light (WL) brightness, both after three-dimensional Fourier "cone" filtering passing only modulatory phenomena with horizontal speeds below $6 \mathrm{~km} \mathrm{~s}^{-1}$. In both image sequences this filtering removes the acoustic oscillations. In the WL image sequences the filtering isolates the granulation; in the UV image sequences it isolates the slowly evolving background mesh pattern seen also in Ca II K filtergrams (Lites et al. 1999) on which $\mathrm{K}_{2 V}$ internetwork grains are superimposed (Cram \& Damé 1983). The resulting low-pass UV against low-pass WL sequence shows a weak but long-lived anticorrelation, implying that there is a weak tendency for bright UV background to be located above intergranular lanes. This correlation would identify the background as "reversed granulation" if it were more pronounced (moment curves aligned along the backwards diagonal) and more instantaneous. The weakness of the correlation, its initial increase with increasing delay, and its long persistence indicate something else than reversed granulation.

The remaining three columns of Fig. 4 are time-delay scatter sequences for unfiltered UV, low-pass UV and high-pass UV against low-pass UV. The second column shows high initial correlation with $2: 1$ slope, implying that peaks in 


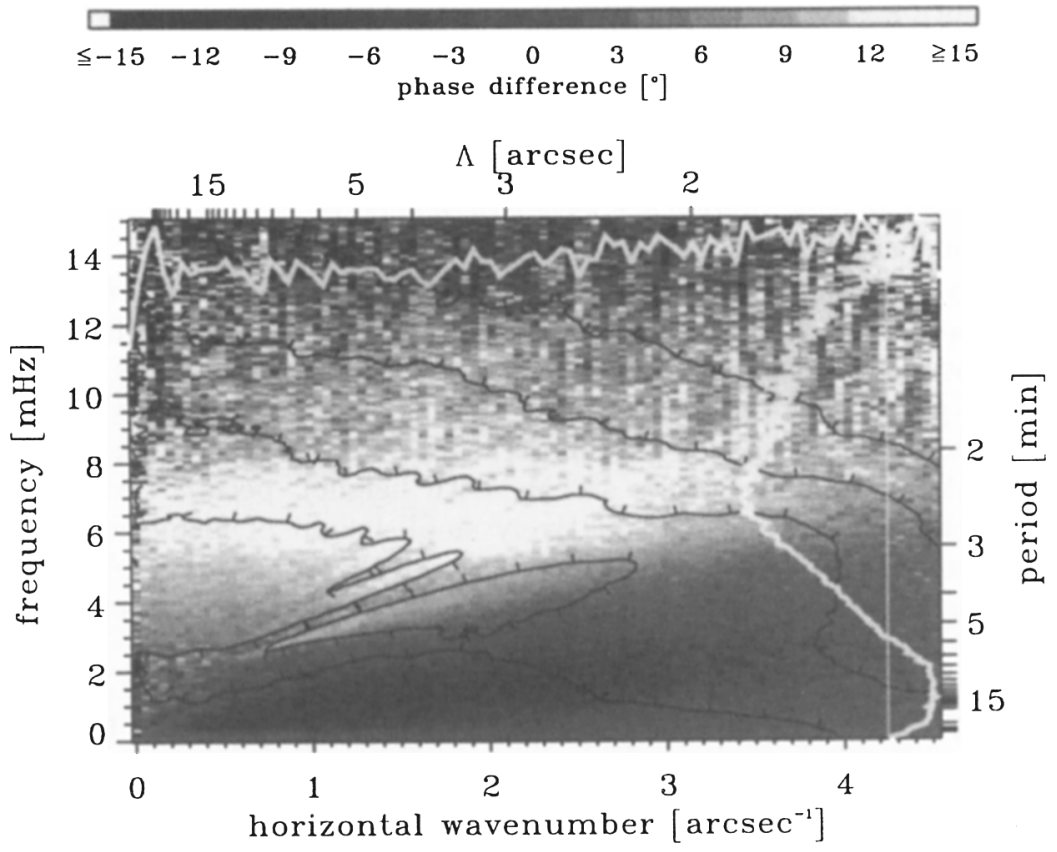

Figure 5. Upper-photosphere $\left(k_{h}, f\right)$ phase difference spectrum from TRACE $1700 \AA$ and $1600 \AA$ images. Format as for Fig. 3. Greyscale: 1700-1600 $\AA$ phase difference as specified in the bar above the plot. The contours specify $1700-1600 \AA$ coherence at values $C=0.4,0.6$, 0.8 and 0.95 with the ticks directed to lower values. From Rutten \& Krijger (2002).

internetwork UV brightness occur preferentially when also the slowly-changing background peaks in brightness. The latter contributes about half of the total peak brightness. Thus, the background is an important co-localization agent in the occurrence of internetwork grains.

The third column of Fig. 4 is an autocorrelation sequence which shows that the UV background has about five-minute lifetime at a given spatial position.

The final column of Fig. 4 plots scatter correlations between the threeminute oscillation amplitude and the background amplitude. The slightly curved shape of the vertical moment curves in the first three panels implies that large three-minute excursions of either sign tend to correlate slightly with large background amplitude, i.e., act partially (but not strongly) as a modulation signal.

If the slowly changing UV background pattern is not reversed granulation nor acoustic, it has to be something else. Figure 5 supplies the answer. It shows $\left(k_{h}, f\right)$ Fourier decomposition for the $\Delta \phi(1700-1600)$ brightness modulation phase differences, determined from simultaneous $1700 \AA$ and $1600 \AA$ image sequences in the same TRACE data set as used in Figs. 3-4. It shows phase contrast along the pseudo-ridges which is discussed in more detail in Krijger et al. 
(2001). What is important here is the dark wedge of negative phase under the Lamb line, with high coherence. The wedge location, its Lamb-line delimitation and its negativity identify gravity waves as cause. The high coherence indicates that it corresponds to most of the high power in this location in Fig. 3. Thus, the slowly changing UV background is made up of gravity waves.

\section{Discussion}

For discussion of various obvious issues (scales of the apparent gravity wave pattern, the role of internetwork magnetism, the penetration of gravity waves up to or above magnetic canopies) I refer the reader to the discussion in Rutten \& Krijger (2002). Here, I want to add two speculations.

The first one concerns the possible presence of global $g$-mode signatures in the gravity waves diagnosed here. I call the latter "atmospheric gravity waves" because they are seen in the solar atmosphere and on the assumption that they fully result from convective overshoot at the solar surface, i.e., when granular upwellings at the top of the convection zone penetrate into the overlying domain of convective stability caused by large photon losses in the optical continuum. However, it cannot be excluded that some of the gravity wave signal detected here arises from comparable downward overshoot at the bottom of the convection zone. Such internal waves might have global persistence, suffer cavity mode selection, lift the whole convection zone and increase in thermal amplitude while propagating up the $1700 \AA$ response height. Ultraviolet brightness sequences may provide a less noisy diagnostic for such gravity waves on near-granular scales than brightness or even Dopplershift sampling of the granule-infested low photosphere. The helioseismological measurements at the South Pole during the past decades sampling Ca II K brightness addressed larger scales. Thus, continuous long-duration full-disk high-resolution imaging of the upper photosphere in the ultraviolet, as will become feasible with the planned Solar Diagnostics Observatory, may represent the best way to search for global high- $l g$-modes. A long shot but perhaps worth pursuing.

The second speculation concerns the nature of the "basal flux" which describes a ubiquitous contribution to the surface radiation from cool stars observed in the CaII $\mathrm{H} \& \mathrm{~K}$ lines and other activity indicators underlying the varying amount of emission added by magnetic activity (e.g., Schrijver 1995). The solar results summarized here indicate that this basal emission is produced from acoustic waves and gravity waves together, in roughly fifty-fifty division. Acoustic heating theories that aim to reproduce the observed basal flux as in the modeling of Cuntz et al. (1999) need to take the additional, presumably non-heating, gravity-wave ingredient into account.

\section{Conclusions}

The upper photospheres of cool stars are now amenable to detailed hydrodynamical modeling including wave generation and wave interference. Numerical simulation codes that correctly include convective collapse and overshoot as well as acoustic waves and shocks and internal gravity waves should do the job to 
some extent, limited by the actual presence of reflecting and mode-converting magnetic canopies at least in the solar case.

Acoustic excitation by extreme acoustic flux events takes place in noticeable but non-dominant fashion.

Atmospheric gravity waves contribute about half the amplitude of the internetwork brightness modulation observed in Ca II K H \& K or ultraviolet continua including $\mathrm{K}_{2 V}$ and ultraviolet internetwork grains. They are likely to also contribute half of the cool-star basal flux in low-chromosphere activity indicators.

The gravity-wave interference pattern may contain a global $g$-mode contribution excited by overshoot at the bottom rather than the top of the convection zone, but present data show no indication that this is the case.

Acknowledgements. I thank the organizers for inviting and enabling me to speak, acknowledge inspiring discussions about gravity waves with Robert F. Stein on the roundabout way to Uppsala, and apologize to Alan M. Title for inserting Fig. 1 in this writeup.

\section{References}

Bonet J. A., Marquez I., Vázquez M., Wöhl H., 1991, A\&A 244, 492

Brown T. M., Harrison R. L., 1980, ApJ 236, L169

Carlsson M., Stein R. F., 1997, ApJ 481, 500

Cram L. E., 1978, A\&A 70, 345

Cram L. E., Damé L., 1983, ApJ 272, 355

Cuntz M., Rammacher W., Ulmschneider P., Musielak Z. E., Saar S. H., 1999, ApJ 522, 1053

Deubner F.-L., Fleck B., 1989, A\&A 213, 423

Durrant C. J., Nesis A., 1981, A\&A 95, 221

Frazier E. N., 1968, ApJ 152, 557

Goode P. R., 2002, American Astronomical Society Meeting 200, 5305

Goode P. R., Gough D., Kosovichev A. G., 1992, ApJ 387, 707

Goode P. R., Strous L. H., Rimmele T. R., Stebbins R. T., 1998, ApJ 495, L27

Hoekzema N. M., Rimmele T. R., Rutten R. J., 2002, A\&A 390, 681

Hoekzema N. M., Rutten R. J., Brandt P. N., Shine R. A., 1998, A\&A 329, 276

Kneer F., von Uexküll M., 1993, A\&A 274, 584

Komm R., Mattig W., Nesis A., 1991, A\&A 252, 827

Krijger J. M., Rutten R. J., Lites B. W., Straus T., Shine R. A., Tarbell T. D., 2001, A\&A 379, 1052

Lighthill M. J., 1967, in R. N. Thomas (ed.), Aerodynamical Phenomena in Stellar Atmospheres, IAU Symp. 28, Academic Press, New York, p. 429

Lites B. W., Leka K. D., Skumanich A., Martínez Pillet V., Shimizu T., 1996, ApJ 460, 1019

Lites B. W., Rutten R. J., Berger T. E., 1999, ApJ 517, 1013 
McIntosh S. W., Bogdan T. J., Cally P. S., Carlsson M., Hansteen V. H., Judge P. G., Lites B. W., Peter H., Rosenthal C. S., Tarbell T. D., 2001, ApJ 548, L237

Mihalas B. W., Toomre J., 1981, ApJ 249, 349

Mihalas B. W., Toomre J., 1982, ApJ 263, 386

Rast M. P., 1999, ApJ 524, 462

Restaino S. R., Stebbins R. T., Goode P. R., 1993, ApJ 408, L57

Rimmele T. R., Goode P. R., Harold E., Stebbins R. T., 1995, ApJ 444, L119

Rosenthal C. S., Bogdan T. J., Carlsson M., Dorch S. B. F., Hansteen V., McIntosh S. W., McMurry A., Nordlund A., Stein R. F., 2002, ApJ 564, 508

Rutten R. J., 1999, in B. Schmieder, A. Hofmann, J. Staude (eds.), Magnetic Fields and Oscillations, Procs. Third Adv. in Solar Physics Euroconf., ASP Conf. Ser., Vol. 184, p. 181

Rutten R. J., Krijger J. M., 2002, A\&A submitted

Schmieder B., 1976, Sol. Phys. 47, 435

Schmieder B., 1977, Sol. Phys. 54, 269

Schrijver C., 1995, A\&AR 6, 181

Schrijver C. J., Title A. M., 2002, Sol. Phys. 207, 223

Skartlien R., Stein R. F., Nordlund A., 2000, ApJ 541, 468

Staiger J., 1987, A\&A 175, 263

Staiger J., Mattig W., Schmieder B., Deubner F. L., 1984, Memorie della Societa Astronomica Italiana 55, 147

Stein R. F., 1967, Sol. Phys. 2, 385

Stein R. F., Nordlund Å., 1989, ApJ 342, L95

Stein R. F., Nordlund A., 2000, Sol. Phys. 192, 91

Straus T., Bonaccini D., 1997, A\&A 324, 704

Strous L. H., 1994, Dynamics in Solar Active Regions: Patterns in MagneticFlux Emergence, $\mathrm{PhD}$ thesis, Utrecht University

Strous L. H., Goode P. R., Rimmele T. R., 2000, ApJ 535, 1000

van Ballegooijen A. A., Nisenson P., 1999, in T. Rimmele, K. Balasubramaniam, R. Radick (eds.), High Resolution Solar Physics: Theory, Observations, and Techniques, Procs. 19th NSO/Sacramento Peak Summer Workshop, ASP Conf. Ser.183, p. 30

van Ballegooijen A. A., Nisenson P., Noyes R. W., Löfdahl M. G., Stein R. F., Nordlund A., Krishnakumar V., 1998, ApJ 509, 435

Whitaker W. A., 1963, ApJ 137, 914 